\title{
Minimizing Shirking in Auctions and Tournaments
}

\author{
Chen Cohen ${ }^{1}$, Moshe Schwartz ${ }^{2}$ \\ ${ }^{1}$ Department of Economics, Ashkelon Academic College, Ashkelon, Israel \\ ${ }^{2}$ Department of Sociology and Anthropology, Ashkelon Academic College, Ashkelon, Israel \\ Email: moshesc@gmail.com, chencohe@bgu.ac.il
}

Received April 12, 2013; revised May 12, 2013; accepted June 12, 2013

Copyright (C) 2013 Chen Cohen, Moshe Schwartz. This is an open access article distributed under the Creative Commons Attribution License, which permits unrestricted use, distribution, and reproduction in any medium, provided the original work is properly cited.

\begin{abstract}
The present paper deals with two-player all pay contests in which a tie is due to slacking, showing that to reduce the likelihood of such an occurrence, slackers should be denied any reward, or even punished. The denial of reward, or the punishment, inflicted on substandard performers, may spur some players to bigger efforts, or induce others to avoid contests in which they are unable to meet standards. However denying any reward to those making small but not substandard efforts, would not raise the proportion of those making the maximum effort, while more competitors would abstain from any effort at all, detracting from overall performance. The point allocation rule suggested by this paper is thus shown to improve on its alternatives. The paper proposes changing the rules of point allocation in soccer, to reduce the incidence of non-scoring draws, often the outcome of bad playing or of extreme risk avoidance, expressed in purely defensive game strategies. Under the new rules, a win would award a team with three points, a scoring draw would entitle the teams to one point each, but neither team would receive any points for non-scoring draws. We show that this change would reduce the numbers of games ending in 0:0, while raising the numbers of goals in other games, thus boosting spectator enjoyment.
\end{abstract}

Keywords: Tournaments; Auctions; Nash Equilibrium; Soccer; Draws; All-Pay-Contests

\section{Introduction}

In winner-takes-all contests, the winner is the one who invested the most in the game and the sole prize receiver, even though all contestants have incurred costs. Some winner-take-all contests are adjudicated through objective valuations, such as tests of skill and ability, or by votes, others-through more subjective valuations. If the best contestants perform the same, the contest ends without either side winning, i.e. with a tie. Ties occur in sports competitions, but also in other contests, such as elections, if a runoff is required between recipients of the largest numbers of votes.

Winner-takes-all formal game theory has been applied to rent-seeking by Hillman and Samet [1] and Hillman and Riley [2]; lobbying by Becker [3] and Che and Gale [4]; R\&D races by Dasgupta [5]; political contests by Snyder [6]; and wars of attrition by O'Neil [7]. Cohen and Sela (CS) [8] modeled the points system in soccer (European football) matches using competitions of the "All Pay" type (evaluating the optimal prize policy in the case of a draw, as against a win).Like CS, we look at the contest output (rather than at the inputs) and deny any prize to low performing teams, even if the teams they have played against have done no better. CS has shown that the players' efforts in equilibrium do not depend on the expected prize in case of a tie, as long as it is one third or less than the prize for winning.

Minimax strategies are compatible with defensive soccer games, ending up in draws, and often frustrating spectators. To reduce the likelihood of draws, it has been suggested to lessen their relative reward, by increasing pay-offs for wins. This has led to the 3-1-0 (the three point victory) system. Moreover, it has been demonstrated that a 4-1-0 or 5-1-0 rule, though intuitively attractive, would not improve results [8]. However, Broca and Carillo [9] have also shown that under some conditions, the 3-1-0 rule is counterproductive, inducing players to play more defensively than under $2 \mathrm{PV}$ (to avoid being led early on in the match). Another discussed improvement is the GG (Golden Goal) rule, whereby when two teams are tied at the end of the regular time, the first to score within the 30 minutes of overtime, wins the match. Thus the GG rule reduces play time, driving up the likelihood of offensive strategies in the second half of the game. Broca and Carillo [9] have also shown that combining the GG rule and the 3-1-0 system leads to 
better results than 3-1-0 alone.

Statistical analysis vindicates the decision of the English Football League, in 1982, to award three points for a win, instead of the previous two points (thus reducing the prize for a draw to one third of that for a win). Indeed, following the League's decision, the average number of goals scored in its' matches increased dramatically Dobson and Goddard [10]. Moschini [11] has used a data set spanning 30 years and 35 countries, showing that the system has achieved its purpose (even without being augmented by the GG rule).

The original contribution of the present paper, henceforth CSI (Cohen and Schwartz Improvement), is reducing the likelihood of ties due to lack of a serious effort to win on the part of the contestants. We show that to achieve this purpose, slacking teams and those who have played a purely defensive game, failing to take any risks, should be denied any reward whatsoever. This denial of reward inflicted on substandard performers, may spur some teams to bigger efforts, while inducing others to avoid contests if they are unwilling or unable to meet minimal standards.

However if we denied any reward to those make small but not substandard efforts (Overextended CSI rule, henceforth OCSI), more competitors would abstain from any effort at all, while the numbers of those making the maximum effort would not increase. Thus the CSI rule, suggested by this paper, is shown to improve on both CS and OCSI.

In the present paper, we show that the CSI model, which adds to the CS model the denial of any points to the teams involved in scoreless draws, will reduce their frequency. We also show that the proposed change increases the average number of goals per match.

Part 2 of this paper presents the model and its Nash equilibrium point. Part 3 analyzes the results, offers examples and presents conclusions.

\section{The Model and the Nash Equilibrium Point}

Let us assume that two players are vying for one prize in a one-stage all-pay contest. Each player invests $x_{i} \in\{1,2,3, \cdots\}$. The investment includes money, time, human capital and physical efforts (depending on competition type-as listed above), as well as the energy expended in the actual competition. Every player shoulders such costs, irrespective of whether he ends up by winning or losing: in such a game, the one investing the most, wins. We assume complete information and a symmetrical model, in which winning the competition, carries the same value $V$ for both players. In case of a draw where both players invested equal efforts, they will each have a $1 / 3$ probability of winning the prize.
To allow positive probability for a draw, we assume that the number of possible strategies is finite. Thus we have to use a finite game theory model. The competition planner must determine the prize, not only for a win, but also for a draw, with a view to ensuring that in the latter case the probable prize is less than half the prize in a win.

\subsection{The CS Model}

According to this model, a draw gains one point for each of the participating teams, whereas a win gains three points for the victorious team.

The payment functions per team in this model are:

$$
u_{i}= \begin{cases}-x_{i} & \text { if } x_{i}<x_{j} \\ \frac{1}{3} v_{i}-x_{i} & \text { if } x_{i}=x_{j} \neq 0 \\ v_{i}-x_{i} & \text { if } x_{i}>x_{j}\end{cases}
$$

\subsection{The CSI Model}

According to this model, a draw gains one point for each of the participating teams, except if it is a 0:0 draw; in this later case no team gets any points. A win gains three points for the victorious team.

The payment function per team in this model are:

$$
u_{i}= \begin{cases}-x_{i} & \text { if } x_{i}<x_{j} \\ \frac{1}{3} v_{i}-x_{i} & \text { if } x_{i}=x_{j} \neq 0 \\ 0 & \text { if } x_{i}=x_{j}=0 \\ v_{i}-x_{i} & \text { if } x_{i}>x_{j}\end{cases}
$$

Considering the payment function for teams, one may observe that any team invests an effort to win (all pay), which implies that if it loses the game or ends up with $0: 0$, it has incurred a negative gain, as its investment has yielded no fruit.

\subsection{The OCSI Model}

This model fails to achieve an additional improvement in point allocation. To the contrary, it worsens results.

The model awards one point for draws, except for 0:0 and 1:1 draws. In the event of a win, the winning team gets three points and the losing one-none.

The payment function in this model is:

$$
u_{i}= \begin{cases}-x_{i} & \text { if } x_{i}<x_{j} \\ \frac{1}{3} v_{i}-x_{i} & \text { if } x_{i}=x_{j} \neq 0,1 \\ 0 & \text { if } x_{i}=x_{j}=0 \text { and } x_{i}=x_{j}=1 \\ v_{i}-x_{i} & \text { if } x_{i}>x_{j}\end{cases}
$$




\subsection{Equilibrium}

In the CSI model, an equal equilibrium of pure strategies cannot exist, since, when player $i$ invests output $x_{1}$ and $V$ $>x_{1}$, there is an incentive for player $j$ to invest one unit more than $x_{1}$. Similarly, for player $i$ there will now be an incentive to invest more than player $j$ and so on, up to value $V$, at which point an additional investment will be not be worthwhile for either player. However, in such a game, a Nash equilibrium point may exist for mixed strategies, which means that one can invest effort with a given probability in the original game, while maintaining the likelihood of equal gain for any strategy employed.

Proposition 1: Consider two players with the same prize valuation $V$, who compete in an all-pay contest for a unique prize. If the rules are those of the CSI model, there is a symmetric equilibrium in which any given player chooses any given effort $x \in\{1, \cdots, v-1\}$ with the probability $P_{V-i}=\frac{2^{i}+(-1)^{i+1}}{2^{i} V}$, while his probability of investing no effort is $P_{0}=\frac{2^{V}+(-1)^{V+1}}{2^{V-1} \cdot 3 V}$.

Equilibrium in a mixed strategy is derived from assumption that every effort assigned a positive probability provides the player (in our case the individual team) with the same expected gain. Thus the probabilities below are the derivative of $V$.

Equations showing the equal probability of winning with any strategy: the equations have the form:

$$
V_{i} \cdot(\text { prob' of winning })-x_{i}=k
$$

where $k$ is the expected payoff

An additional equation shows that the sum of probabilities is equal to 1 .

$$
\sum_{i=0}^{V-1} p_{i}=1
$$

Examples of equilibrium for values other than $V$ can be found in Appendix 1.

Proposition 2: Consider two players with the same prize valuation $V$ who compete in all-pay contest for a unique prize. The expectancy of goals in the CSI model is larger then the expectancy in the original CS model.

Proof. In the CSI model the expectancy of goals per player per match is:

$$
\sum_{i=1}^{V-1} p_{i} \cdot i=\sum_{i=1}^{V-1}\left[\frac{2^{i}+(-1)^{i+1}}{2^{i} \cdot V}\right] \cdot i
$$

while this expectancy, according to the original CS model is:

$$
\sum_{i=1}^{V-1} \frac{1}{V} \cdot i=\frac{V-1}{2}
$$

The CSI model always yields a larger expectancy than the original one:

$$
\begin{aligned}
& \sum_{i=1}^{V-1} p_{i} \cdot i-\sum_{i}^{V-1} \frac{1}{V} \cdot i \\
& =\sum_{i=1}^{V-1}\left[\frac{2^{i}+(-1)^{i+1}}{2^{i} \cdot V}\right] \cdot i-\sum_{i=1}^{V-1} \frac{1}{V} \cdot i \\
& =\sum_{i=1}^{V-1} \frac{(-1)^{i+1}}{2^{i} \cdot V}>0
\end{aligned}
$$

Thus, the expected output is likely to rise, due to the proposed change.

However, denying any reward to those attaining 1:1 ties as well as to those attaining 0:0 ties, would not improve the results but rather make them worse (OCSI Model). In such a case, the payment function for the player is:

$$
u_{i}= \begin{cases}-x_{i} & \text { if } x_{i}<x_{j} \\ \frac{1}{3} v_{i}-x_{i} & \text { if } x_{i}=x_{j} \neq 0,1 \\ 0 & \text { if } x_{i}=x_{j}=0 \text { and } x_{i}=x_{j}=1 \\ v_{i}-x_{i} & \text { if } x_{i}>x_{j}\end{cases}
$$

Proposition 3: Consider two players with the same prize valuation $V$ who compete in all-pay contest for a unique prize. If the rules are those of the overextended ISC model, then, there is symmetric equilibrium in which any given player chooses every effort $x \in\{2, \cdots, v-1\}$ with the probability $P_{V-i}=\frac{2^{i}+(-1)^{i+1}}{2^{i} V}$, and choose to invest effort in level 1 with the probability $P_{1}=1-\left[\frac{1}{V}+\sum_{i=1}^{V-2}\left(\frac{2^{i}+(-1)^{i+1}}{2^{i} V}\right)\right]$ and invest no effort in the probability $P_{0}=\frac{1}{V}$ for every $V-i>0$, when $i$ is a positive integer.

The above equilibrium can also be derived from the equation system representing the equal expectancy of gain for any investment strategy with a positive probability between very small and $V-1$

$$
V_{i} \cdot(\text { prob' of winning })-x_{i}=k
$$

With $k$ as the expected payoff

An additional equation shows that the sum of probabilities is equal to 1 .

$$
\sum_{i=0}^{V-1} p_{i}=1
$$

From the above equation systems we derive the given probabilities at equilibrium: 


\subsection{Comparing CS, CSI and OCSI}

\begin{tabular}{|c|c|c|c|c|c|c|c|}
\hline$V=5$ & $P_{0}$ & $P_{1}$ & $P_{2}$ & $P_{3}$ & $P_{4}$ & $P_{5}$ & T.E \\
\hline \multirow[t]{2}{*}{ CS } & 1 & 1 & 1 & 1 & 1 & & \multirow{2}{*}{2} \\
\hline & $\overline{5}$ & $\overline{5}$ & $\overline{5}$ & $\overline{5}$ & $\overline{5}$ & & \\
\hline \multirow{2}{*}{$\begin{array}{l}\text { CSI (Cohen and } \\
\text { Schwartz } \\
\text { Improvement) }\end{array}$} & 11 & 15 & 18 & 12 & 24 & & \multirow{2}{*}{2.28} \\
\hline & 80 & 80 & 80 & 80 & 80 & & \\
\hline \multirow{2}{*}{$\begin{array}{l}\text { OCSI } \\
\text { overextended CSI } \\
\text { model }\end{array}$} & 16 & 10 & 18 & 12 & 24 & & \multirow{2}{*}{2.22} \\
\hline & $\overline{80}$ & $\overline{80}$ & $\overline{80}$ & $\overline{80}$ & $\overline{80}$ & & \\
\hline
\end{tabular}

Allocating 0 points for a 1:1 draw (in addition to 0 points for a 0:0 draw) does not spur the players to make additional efforts but rather discourages them. Those who made no efforts in the CSI case, will keep abstaining from them, while those who made a small effort, will desist from it. Meanwhile, the proportion of those making a serious effort will not change.

\section{Analysis and Conclusions}

Requiring a minimum entrance price (in an auction or in other contests) under conditions of incomplete information, or imposing a substantial fine on contestants who fail to comply with what they committed to do (deliver goods, do a job, or stick to the tender if they win, rather than "coping out" at the last minute), is only effective if matters are clear cut (either the contestant stuck to his commitment or he did not). However, sometimes the inputs required in the contest are multiple, complex, and not directly observable. Thus, it is not immediately clear whether enough effort has been invested, which requires a more sophisticated tool, to induce the players to invest at least the minimum. The tool we propose looks at the output of the contest (rather than at the inputs) and denies any prize to players who have demonstrated low performance, even if others have done no better.

Like the original CS model, our CSI model (Cohen and Schwartz Improvement) adds realism without fostering complication. It adds realism, by using the all pay assumption, which implies that players make efforts, whether they end up by winning, losing, or with a draw, and thus these efforts are to be viewed as sunken costs. Still, our model allows for the possibility of investing less then the minimum acceptable effort. However, such an occurrence becomes less likely. In addition, we show that with the new change in decision rule, the likelihood of a player's investing less than the minimal acceptable effort, is below the likelihood of his producing any other output and thus less than the average probability, $1 / V$.

We show that the amended decision rule reduces the frequency of cases in which both players invest less than the acceptable minimum. Thus, the planner wishing to maximize players' investment in the competition, will achieve better results with the proposed decision rule, as it causes a participant investing below the minimal level to lose the potential prize, irrespective of how much his competitor has invested. Our proposal lessens the likelihood of situations in which two competitors, both of whom failed to invest the minimum, still manage to share the spoils between them.

We also show that the amended decision rule improves over both CS and OCSI, as raising beyond that minimum the effort required for a prize (the overextended ISC model) lowers overall game performance, causing those willing to make a small effort to desist from it, without raising the share of those making a serious effort.

\section{REFERENCES}

[1] G. Becker, "A Theory of Competition among Pressure Groups for Political Influence,” Quarterly Journal of Economics, Vol. 98, No. 3, 1983, pp. 371-400. doi: $10.2307 / 1886017$

[2] I. Broca and J. D. Carrillo, "Do the Three-Point Victory and Golden Goal Rules Make Soccer More Exciting?" Journal of Sports Economics, Vol. 5, No. 2, 2004, pp. 169-185. doi:10.1177/1527002503257207

[3] Y. K. Che and I. Gale, "Caps on Political Lobbying," American Economic Review, Vol. 88, No. 3, 1998, pp. 643-651.

[4] C. Cohen and A. Sela, "Contests with Ties," The B.E. Journal of Theoretical Economics, Vol. 7, No. 1, 2007, Article 43.

[5] P. Dasgupta, "The Theory of Technological Competition,” In: J. Stiglitz and G. F. Mathewson, Eds., New Developments in the Analysis of Market Structure, MIT Press, Cambridge, 1986, pp. 519-545.

[6] S. Dobson and J. Goddard, "The Economics of Football," Cambridge University Press, Cambridge, 2001. doi:10.1017/CBO9780511493225

[7] A. Hillman and J. G. Riley, "Politically Contestable Rents and Transfers," Economics and Politics, Vol. 1, No. 1, 1989, pp. 17-39. doi:10.1111/j.1468-0343.1989.tb00003.x

[8] A. Hillman and D. Samet, "Dissipation of Contestable Rents by Small Numbers of Contenders,” Public Choice, Vol. 54, No. 1, 1987, pp. 63-82. doi:10.1007/BF00123805

[9] G. Moschini, "Incentives and Outcomes in a Strategic Setting: The 3-Points-for-a-Win System in Soccer," Working Paper \# 08021, Department of Economics Working Paper Series, Iowa State University, 2008.

[10] B. O’Neal, "International Escalation and the Dollar Auction,” Journal of Conflict Resolution, Vol. 30, No. 1, 1968, pp. 33-50. doi:10.1177/0022002786030001003

[11] J. Snyder, "Elections Goals and the Allocation of Campaign Resources,” Econometrica, Vol. 57, No. 3, 1989, pp. 637-660. doi:10.2307/1911056 


\section{Appendix 1}

Numerical examples according to CSI for various V values.

\begin{tabular}{cccccccc}
\hline$V$ & $P_{0}$ & $P_{1}$ & $P_{2}$ & $P_{3}$ & $P_{4}$ & $P_{5}$ & $P_{6}$ \\
\hline 1 & 1 & & & & & & \\
2 & $\frac{1}{4}$ & $\frac{3}{4}$ & $\frac{1}{4}$ & $\frac{2}{4}$ & & & \\
3 & $\frac{1}{4}$ & $\frac{9}{32}$ & $\frac{6}{32}$ & $\frac{12}{32}$ & & & \\
4 & $\frac{5}{32}$ & $\frac{15}{80}$ & $\frac{18}{80}$ & $\frac{12}{80}$ & $\frac{24}{80}$ & & \\
5 & $\frac{11}{80}$ & $\frac{11}{64}$ & $\frac{10}{64}$ & $\frac{12}{64}$ & $\frac{8}{64}$ & $\frac{16}{64}$ & \\
6 & $\frac{7}{64}$ & $\frac{63}{448}$ & $\frac{66}{448}$ & $\frac{60}{448}$ & $\frac{72}{448}$ & $\frac{48}{448}$ & $\frac{96}{448}$ \\
7 & $\frac{43}{448}$ & & & & & & \\
\hline
\end{tabular}

